\title{
A fully packaged self-powered sensor based on near-field electrospun arrays of poly(vinylidene fluoride) nano/micro fibers
}

\author{
Y.-K. Fuh", S.-Ch. Li, Ch.-Y. Chen, Ch.-Y. Tsai \\ Department of Mechanical Engineering, National Central University, No.300, Jhongda Rd., Jhongli District, Taoyuan City \\ 32001, Taiwan (R.O.C.)
}

Received 10 July 2017; accepted in revised form 25 September 2017

\begin{abstract}
Energy harvesting devices based on the triboelectric and piezoelectric principles have been widely developed to scavenge wasteful and tiny mechanical energy into usable electrical energy. In particular, triboelectric energy harvesting generators with relatively simpler structure and piezoelectric fiber-based counterpart with extremely light weight compositions showed a very promising application in the self-powered sensors. In this paper, a novel hybridization of graphenebased piezoelectric generator (GBPG) and graphene-PET triboelectric generator (GPTG) were simultaneously packaged. The integrated structure, graphene-based hybridized self-powered sensor (GHSPS), was demonstrated to be optically transparent and mechanically robust. For the piezoelectrically harvesting device, an in-situ poling and direct-write near-field electrospinning (NFES) Poly(vinylidene fluoride) (PVDF) piezoelectric fibers were fabricated and integrated with a single layer chemical vapor deposition (CVD) grown graphene. On the other hand for GPTG counterpart, two composite layers of a single layer graphene/PET simultaneously served as triboelectrically rubbing layers as well as bottom/top electrode. This GHSPS successfully superimposed both piezoelectric and triboelectric electricity and the synergistically higher output voltage/current/power were measured as $\sim 6 \mathrm{~V} / 280 \mathrm{nA} / 172 \mathrm{nW}$ in one press-and-release cycle of finger induced motion. The proposed GHSPS showed a promising application in the field of self-powered sensors to be ubiquitously implemented in the future Industry 4.0 scenarios.
\end{abstract}

Keywords: smart polymers, electrical properties, poly(vinylidene fluoride) (PVDF), near-field electrospinning (NFES), graphene-based piezoelectric generator $(G B P G)$

\section{Introduction}

Graphene is a two-dimensional (2D) material with 'aromatic' monolayer of $\mathrm{sp}^{2}$ carbon configuration, atomically arranged in a honeycomb and hexagonal lattice. Several distinctive features include ultra-high electron mobility (as high as $26000 \mathrm{~cm}^{2} \cdot \mathrm{V}^{-1} \cdot \mathrm{s}^{-1}$ ), excellent optical transparency ( $\sim 97 \%)$, high mechanical elasticity ( $\sim 1 \mathrm{TPa}$ elastic modulus), and ballistic have charged carrier transport [1-3]. By means of these multi-functional properties, graphene has been actively adopted as an alternative for mechanically brittle transparent conductive oxides (TCOs) [4]. So far, chemical vapor deposition (CVD) growth of graphene has been considered the standard method to make the graphene in a manner of large-area growth with high electron conductivity [5-7]. Other promising applications of graphene were primarily based on its natural chemical inertness and high optical transparency as a transparent electrodes such that wearable electronics and optoelectronics can be fulfilled in the functionalities of flexibility, stretchability and foldability. Previously, graphene and related applications have also been demonstrated for energy harvesting generators due to the increasing interest 
in next-generation flexible electronics and self-powered systems [8-10].

On the other hand, energy harvesting nanogenerators (NGs) have been intensively investigated mainly for realizing the self-powered electronics such as motion monitoring sensors, mobile electronics and implanted medical devices [11-15]. Accordingly, a variety of methods have been demonstrated to convert the mechanical energy into the electric energy using different energy transduction mechanisms such as piezoelectricity, triboelectricity, thermoelectricity, electromagneticity [16]. For example, piezoelectric materials can provide a feasible way to effectively harvest energy from human actions or ambient sources [1719] rather than solely depending on commercial cell batteries. The first NG was previously proposed with the one-dimensional zinc oxide $(\mathrm{ZnO})$ nanowires (NWs) [20]. In particular, PVDF was a polymeric and relatively simply fabricated piezoelectric material with electromechanical coupling of a great piezoelectric constant $\left(d_{33} \sim 57.6 \mathrm{pm} / \mathrm{V}\right)$ [21]. Typical method for making the PVDF micro-nano fibers (MNFs) was utilizing the near-field electrospinning technique [22]. In-situ poled and highly aligned MNFs can be fabricated directly on the flexible substrates without complicated treatments [23]. On the contrary, previous works regarding using normal electrospinning to prepare PVDF nanofibers had been reported. For example, flexible PVDF/PI nanofiberreinforced composites were fabricated by layer-bylayer hot-pressing the random PVDF nanofibers and aligned co-PI nanofibers such that high mechanical strength and good dielectric properties can be obtained for modern electronics [24]. Furthermore, concerning another piezoelectric material of energy harvesting system, nanocomposites with piezoelectric $\mathrm{BaTiO}_{3}$ fillers has been investigated. For example, PVDF copolymer vinylidene fluoride-trifluoroethylene and composites of the $\mathrm{BaTiO}_{3}$ has been investigated and it was found that the best energy harvesting performance is 0.03 and $25 \mu \mathrm{W}$ under low and high mechanical deformations [25]. The piezoelectric $\mathrm{BaTiO}_{3}$ have been investigated as a function of ceramic filler content such that the larger filler average sizes, the generated power decreases for filler contents above $5 \%$ due to increase of the mechanical stiffness [26]. With above-mentioned advantageous characteristics, PVDF piezoelectric MNFs have previously been demonstrated as human motions sensors [27]. A variety of human-induced motions such as walking step, vocal cord vibration, heart beating and muscle movement can be differentially detected [28-31]. On the contrary, the triboelectric NGs have been tremendously developed as in recent years as self-powered, sustainable and green sources for personal devices. Hybridization of these various form of energy harvesting generators included self-powered devices, pyroelectric, and hybrid or triple energy-harvesting were also demonstrated to scavenge the tiny environmental mechanical and thermal energy [32-35]. However, the optically transparent and structurally flexible issues need to be addressed for the application in the self-powered devices. New thrust fields such as wearable skin sensor, touch screen have open up the device requirement of high optical transmittance and mechanical robustness. In this research, rectangular shape of graphene was used as a dual functionality, i.e., piezoelectrically as the current collecting electrode and triboelectrically as the rubbing layer against the graphene/PET layer. The Graphene-based Hybridized Self-Powered Sensor (GHSPS) demonstrated highly transparent and well flexible properties and the output voltage/current were measured as $6 \mathrm{~V} / 280 \mathrm{nA}$ under small mechanical strain of $\sim 0.1$.

\section{Experimental}

Figure 1a provides a schematic illustration of the fabricating processes of a GHSPS used the transparent flexible $3 \times 2 \mathrm{~cm}^{2}$ single layer-graphene. At first, a single layer graphene was initially grown on a $\mathrm{Cu}$ foil via CVD similar to previous study [37]. Next, the well-known wet transfer method was utilized to transfer the single layer graphene onto a flexible PET substrate. To fabricate the graphene-PET triboelectric generator (GPTG), the single layer graphene/PET (bottom side) and the single layer graphene/PET (top electrode) were connected using a plastic spacer of $0.35 \mathrm{~mm}$ thick between the top PET layers and single layer graphene. In order to fabricate the GBPG, the PVDF fibers were fabricated via a direct-write and in-situ poled NFES technique. Approximately 500 PVDF fibers with parallel alignment onto graphene can be fabricated in about $25 \mathrm{~min}$. PDMS ( $\sim 1 \mathrm{~mm}$ thick) was used in the final packaging step to fully encapsulate the composite structures of electrospun fibers, graphene and PET layers. Finally, the GPTG and GBPG can be combined and assembled as an integrated device of GHSPS. PET substrate ( $\sim 0.5 \mathrm{~mm}$ thick). The orange dotted inset in the middle of Figure 1a is shown schematically of the final 

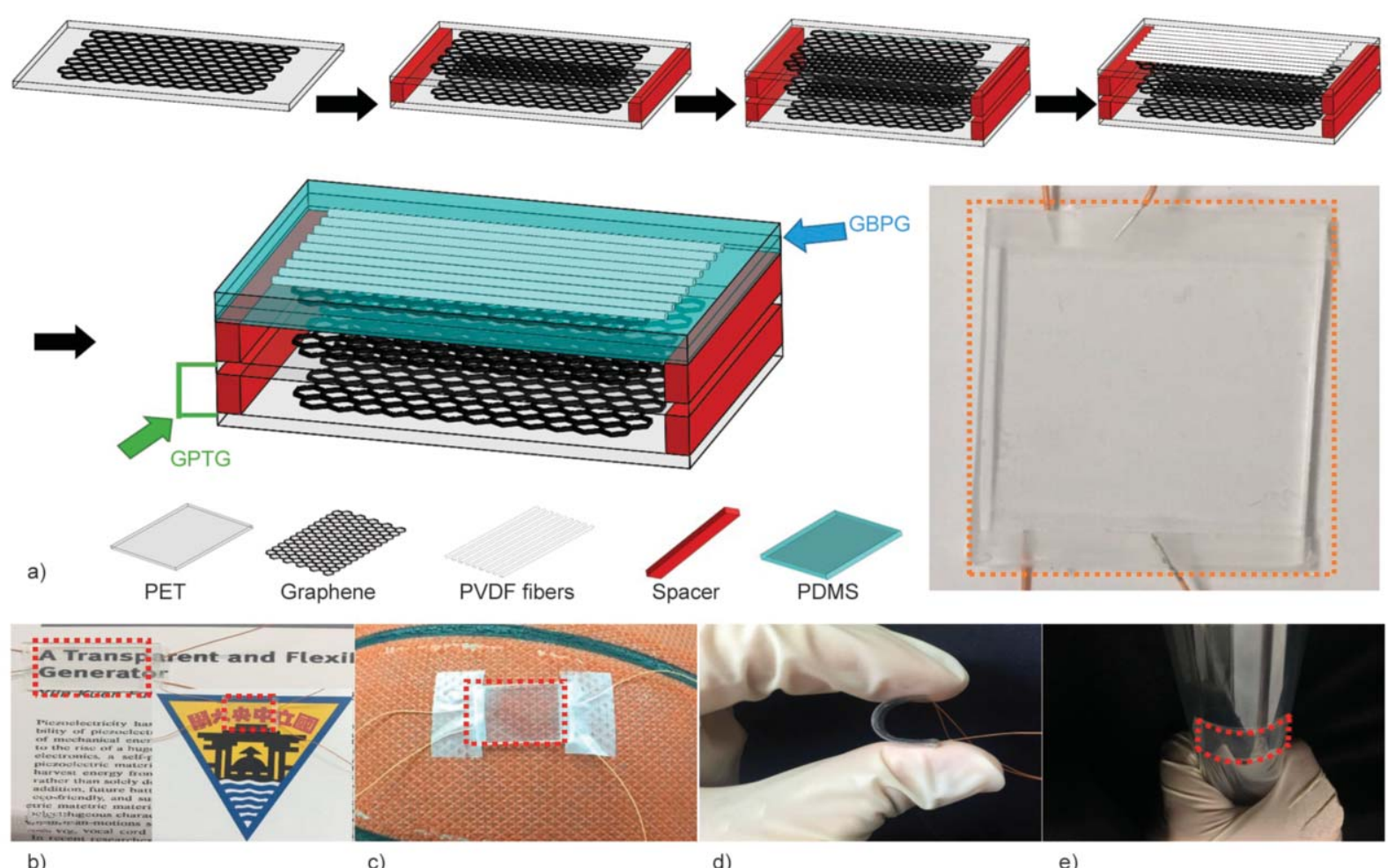

b)

c)

d)

e)

Figure 1. Schematic diagrams of the proposed highly transparent and structurally flexible graphene-based hybridized selfpowered sensor (GHSPS), which is composed of two distinctive structure, i.e., graphene-based piezoelectric generator (GBPG) and graphene-PET triboelectric generator (GPTG) respectively. (a) Structural design and sequential fabrication steps of the GHSPS. Light blue, white, black, gray layers, and red layers correspond to the PDMS, PVDF fibers, graphene, PET substrate, and spacer respectively. The orange dotted inset in the middle shows the fabricated GHSPS. (b) The optical transparency is demonstrated under different background of an academic paper (left) and NCU logo (right). Flexibility of GHSPS which were experimentally tested by (c) adhesively attached the surface of a basketball, (d) finger-folded to a radius of curvature $\sim 1.5 \mathrm{~cm}$ and (e) completely adapted to the rolled-up structure with PET substrate diameter of $\sim 3 \mathrm{~cm}$. The red dotted line shows the GHSPS.

structure of the GHSPS device. The fabrication process of the entire device primarily is composed of two major ocmponents, namely, the piezoelectrical part of electrospun fibers and the triboelectrical part of contacting materials of graphene and PET respectively. Overall, the entire device can be mass produced at a very low-cost and easily scaled up as well as commercialized. In Figure 1b-1e, the wellknown features of graphene, such as its transparent performance, flexibility and compatibility with arbitrary substrates, can be demonstrated. Figure $1 \mathrm{~b}$ shows examples of GHSPS successfully attached to different surfaces, including an academic paper, and NCU logo. Furthermore, Figure 1c-1e shows examples of GHSPS conformally adhere on the curved surface of a basketball, folding to a radius of curvature $\sim 1.5 \mathrm{~cm}$ and adapted to the rolled-up structure with diameter of $3 \mathrm{~cm}$, and the GHSPS are firmly attached to the curved and transparent surface. Finally, the device characterization of SEM, Raman spectra, sheet resistance, transmittance had been carried out to further validate the performance of fabricated GHSPS.

\section{Results and discussion}

For the piezoelectric fiber structures, the GBPG device is mainly consisting of four layers as schematically shown in Figure 2a and can be functionally explained as follows: the single graphene layer serves as the bottom electrode, the PVDF fiber serves as a source of piezoelectric potential, the PET substrate works as the substrate layer which graphene was transferred onto via wet transfer method, and the encapsulated PDMS layer for final packaging layer and a main driving source of strain. Without further complicated post-treatments of electrical and mechanical poling steps, the PVDF fibers can be NFES electrospun procedure in a manner of direct-write, parallel aligned and in-situ poled. Though a mechanical initiation setup to overcome the surface tension of the droplet, PVDF solution was intentionally induced and 


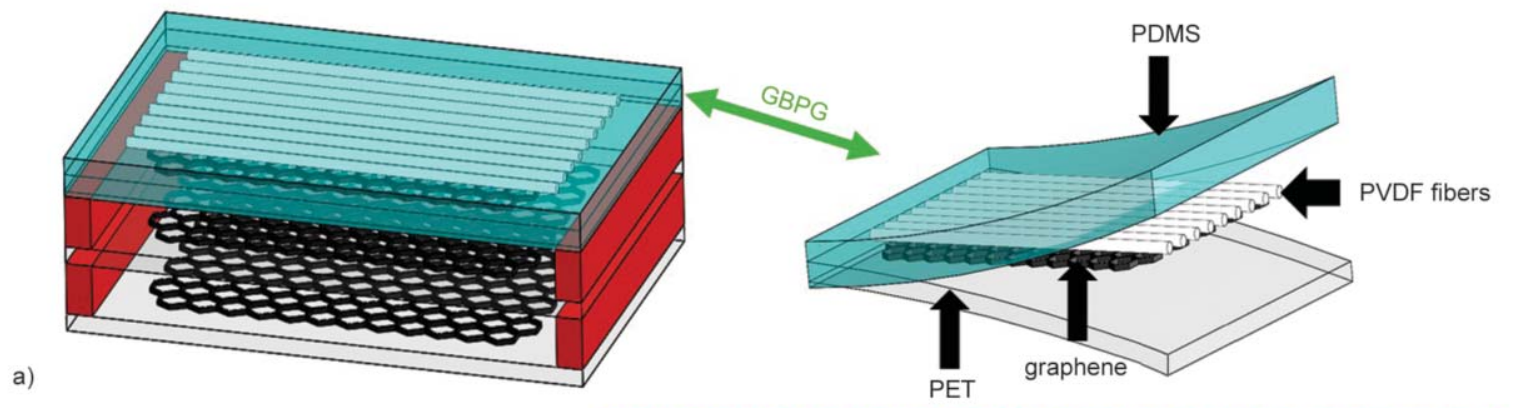

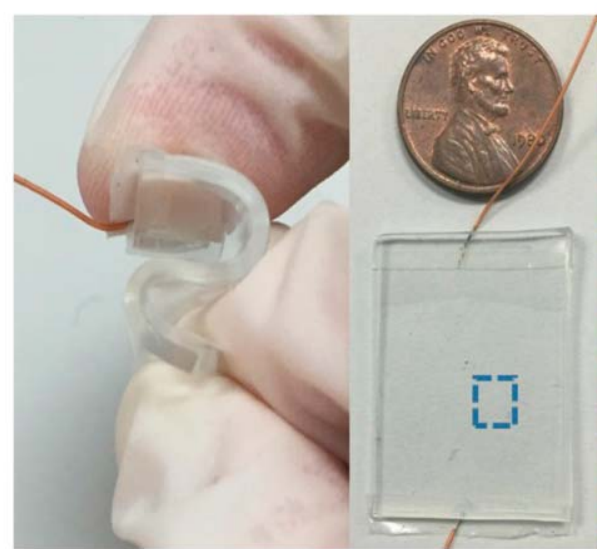

b)

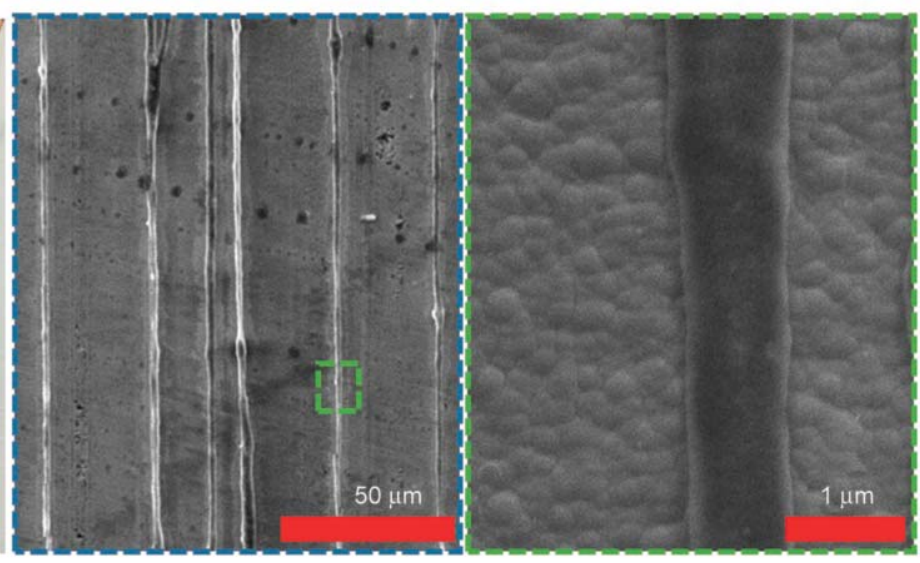

d)

Figure 2. (a) One distinctive feature of the proposed hybridized device is the integrated structure of the graphene electrode and piezoelectric nanofibers array. Light blue, white, black, and gray layers correspond to the PDMS, PVDF fibers, graphene, and PET substrate respectively. (b) Photograph of robustness and flexibility of GBPG device. The size of GBPG was compared with US one cent. Simultaneously rotation and bending will result in a mixed deformed shape of the device. (c) Scanning electron microscope (SEM) photograph of a fabricated device with parallel aligned fibers with diameters in the range of $700 \mathrm{~nm}$ to $2.5 \mu \mathrm{m}$. (d) SEM photograph of a fabricated GBPG with a fiber of diameter $\sim 0.75 \mu \mathrm{m}$.

ejected from the needle tip and a conical shape (Taylor cone) can be formed due to a high electric field. The fine PVDF fibers can be controllably deposited on the graphene electrode and stretched mechanically by setting the $X-Y$ stage motion. In the NFES experiment setup, the distance between graphene electrode and tip of needle was about $1.5 \mathrm{~mm}$ and the applied voltage was $1.2 \mathrm{kV}$. The graphene electrode was initially installed on the $X-Y$ motion stage, which was precisely controlled by a programmed path to deposit the wellaligned the PVDF fibers. Figure $2 b$ shows a photograph of the as-fabricated highly-flexible PVDF GBPG, an ' $S$ ' deformed shape of sequentially double bending can be demonstrated. Figure $2 \mathrm{c}$ shows the SEM photos of as-spun PVDF fibers have diameters ranging from $700 \mathrm{~nm}$ to $2.5 \mu \mathrm{m}$. The diameter of NFES fibers could range from several micro meters to hundred nanometers due to the spin-ability of PVDF solution as identified before [27]. Figure 2d shows an enlarged SEM image of one PVDF fiber, which has a minimum diameter about $0.75 \mu \mathrm{m}$. Due to the use of all polymer layers in our GBPG, the device could be easily bent and released for the selfpowered applications. To demonstrate the potential applications of GBPG solely as the human motion detector, single graphene layer on PET and NFES deposited PVDF fibers can be assembled. Adhesively attached on gloves, a palm movement can be detected and the output voltage and current $1.5 \mathrm{~V} / 150 \mathrm{nA}$ can be measured from the cyclic of grasped/released processes. Furthermore, the authentic piezoelectricity effect of GBPG is validated by measuring the output voltage and current superposition in the serial/parallel configuration. The numerical values of voltage/current for the single GBPG and superimposed GBPGs are experimentally measured as $2.1 \mathrm{~V} / 3.9 \mathrm{~V} / 6.3 \mathrm{~V}$ and $150 \mathrm{nA} / 230 \mathrm{nA} / 390 \mathrm{nA}$.

Figure $3 \mathrm{a}$ shows the fabrication process of the transferred graphene electrode onto PET and the experimental tests of the mechanical properties under extreme conditions. After repeatedly rolling up and releasing the graphene electrode on PET, the graphene electrode remained conductive. The graphene electrode has an excellent mechanical and optical property 
which provides well demonstrations of GHSPS with highly optical transparence and flexibility. To assure the graphene quality, Figure $3 b$ and $3 c$ shows the Raman spectra of single and double layers graphene respectively, as grown via CVD on $\mathrm{Cu}$-foil. It is observed that the well-known $\mathrm{G}$ and $2 \mathrm{D}$ peaks of pristine graphene at $\sim 1584.7$ and $\sim 2690.9 \mathrm{~cm}^{-1}$ respectively. Therefore, the $\mathrm{G}$ and $2 \mathrm{D}$ peaks of both single and double layers graphene can be successfully transferred onto the PET substrates. The observed peaks were at $\sim 1612.1$ and $\sim 2682.7 \mathrm{~cm}^{-1}$ respectively. However, the high transmittance single layer graphene has a relatively higher sheet resistance of $1755 \Omega / \mathrm{sq}$, as compared to sheet resistance of double layers graphene $1401 \Omega /$ sq measured via hall measurement in Figure 3d. Besides, the Micro Spectrometers (OTO Photonics Inc., SE1020) was used to study the transmittance of the two different layer number graphene mount on PET substrates and the well-integrated GHSPS made with single or double layers of graphene electrode. As shown in Figure 3e, the transmittance of single layer graphene is about $95 \%$ and the slightly decreased of two layer graphene of $\sim 93 \%$ transmittance is measured. After integrated with GHSPS devices (structurally composed of single or double layers graphene, an array of piezoelectric PVDF fibers, PET substrates and encapsulated PDMS layer), the fully packaged GHSPS device still exhibited a high transmittance of $\sim 87$ and $82 \%$ in respect to single and double layer graphene based GHSPS. In comparison, previously published transparent electrodes such as the gold nanotrough network sample showed transmittance of $65 \%$ [36]. Furthermore for the fully packaged device as self-powered
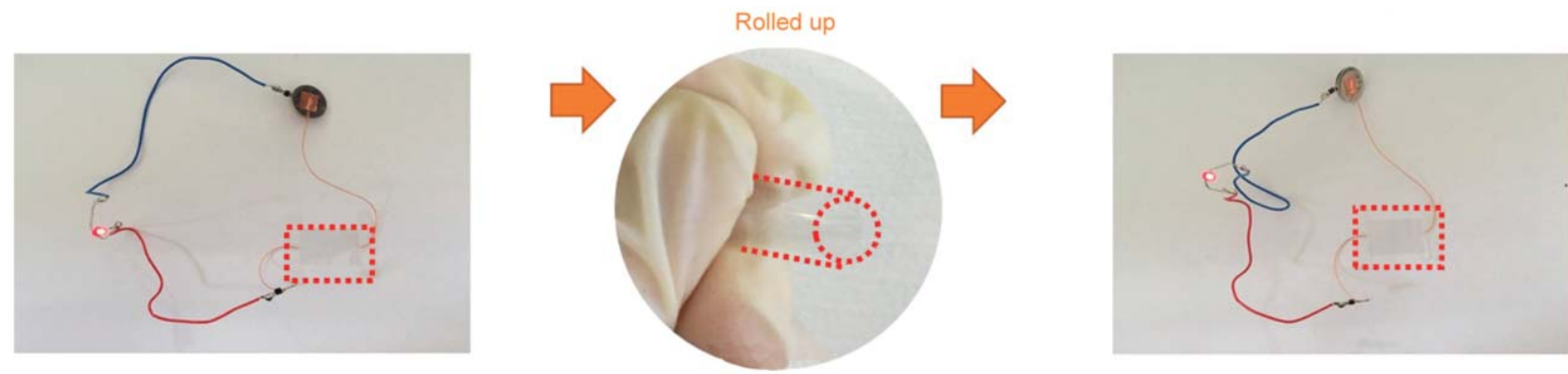

a)
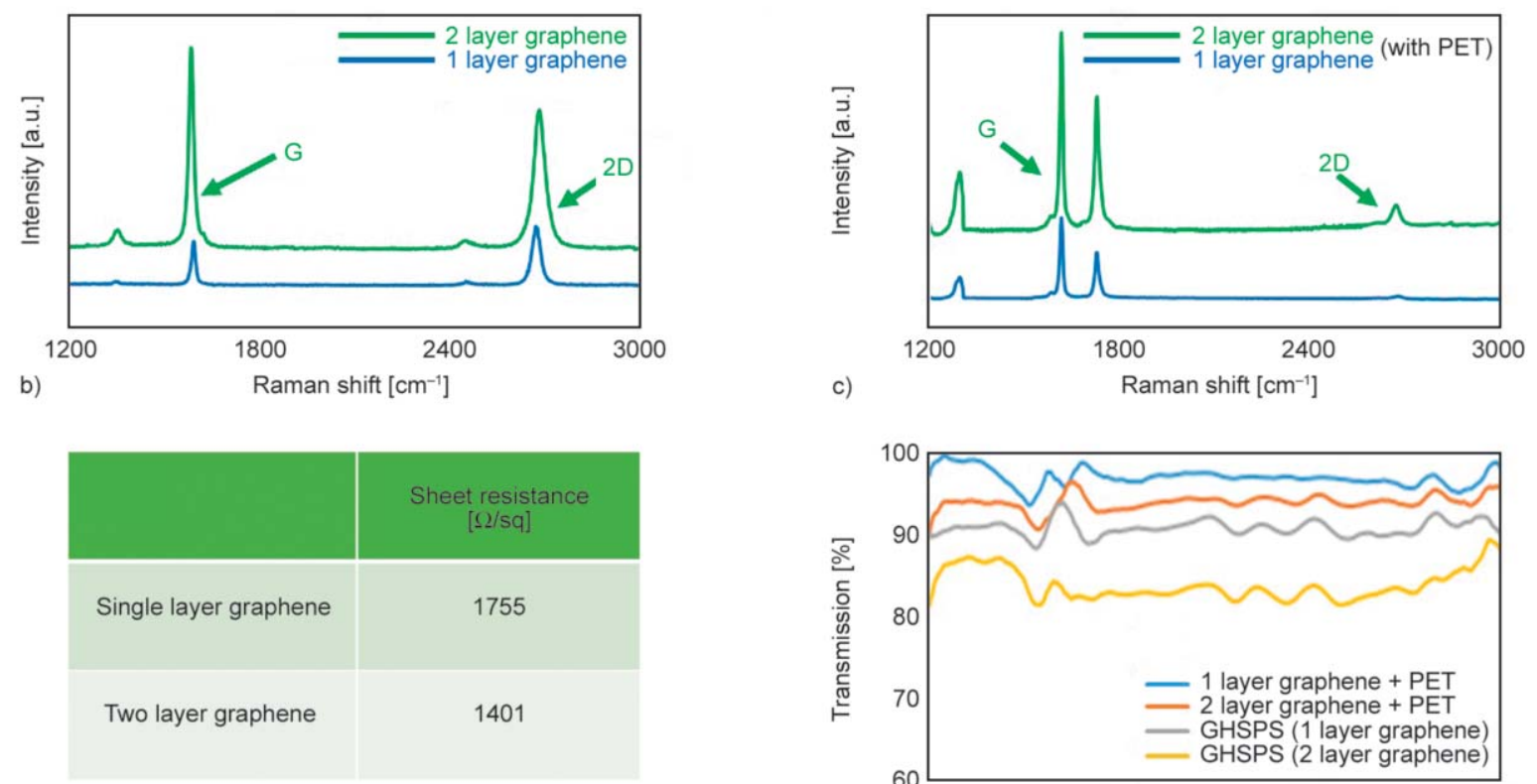

d)

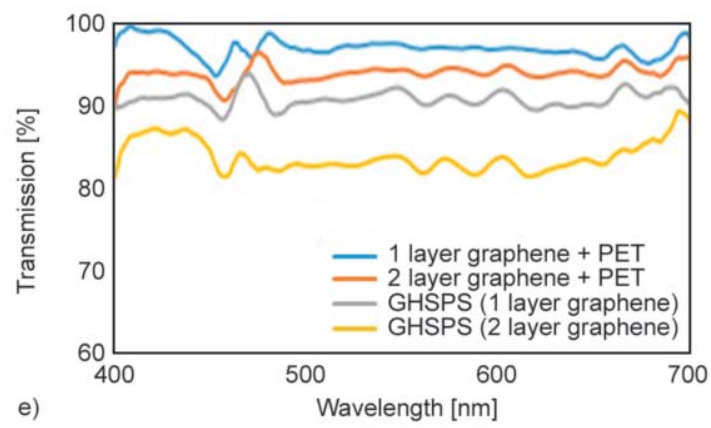

Figure 3. Flexible and transparent graphene electrode and the fabricated GHSPS device. (a) Photographs of electrically conducting PET/graphene composite, fabricated by wet transferring graphene onto PET substrate. After repeatedly rolling up and releasing process, the graphene on PET remained electrically conducting. The G and 2D characteristic peaks were both measured for (b) pristine graphene and (c) graphene on the PET substrate, respectively. (d) Sheet resistance data for single and double layer graphene. (e) The optical transmittance of single and double layer graphene with the PET substrate and the integrated GHSPS devices. 
sensors, the optical transmittance of the nanocomposite films ranged from 71.2 to $75.3 \%$ [32] while the graphene-fibers predecessor exhibited an equivalently high transmittance of $\sim 84$ and $81 \%$ in respect to single and two layer graphene based GPFG.

The working mechanism of the GHSPS is demonstrated in Figure 4a. When an external force was applied on the GHSPS, PVDF fibers are initially induced a piezoelectric potential across the pressed GBPG. The positive and negative charges were generated and accumulated on the opposite direction in order to balance the dipole moment. In the similar fashion, the triboelectric effect can be induced as an external force was applied to the GPTG such that the PET and the single layer graphene are used as the rubbing layer for triboelectric generation. By the physical contact between the PET and graphene layer, the electron injection in the graphene can be produced and triboelectric charges with opposite signs can be generated. As a result, the negatively charged graphene surface and the positively charged PET surface with electrically opposite signs were coincided to incur a relatively small electrostatic potential difference at the interface of PET and graphene. The stronger dipole moment will only be induced after the separation of PET and graphene surfaces, owing to the elastic property of PET film such that a strong electric potential difference is created between the two graphene electrodes. Therefore, the electrostatically accumulated charges were induced the electrons flow from the negatively charged bottom graphene to the positively charged top graphene. The explanation of the electric energy generation process of the GHSPS referred to the work is similar to the previously reported piezoelectric/triboelectric generator [37-38]. Figure $4 \mathrm{~b}$ presents the simulated stress distribution of the integrated GHSPS, both piezoelectric and triboelectric components are included. It was simulated that the symmetrical center of the cross section is experiencing the compressive stress at the push-down stage, with stress values under $5 \mathrm{MPa}$. In Figure $4 c-4 e$, in order to quantitatively analyze the superimposed output of the fabricated GHSPS, an external force was applied to provide the mechanical motion at a frequency of $2 \mathrm{~Hz}$. The open circuit voltage and the short circuit current of the GBPG, GPTG and GHSPS were separately measured. Typically, the average amplitude of the voltage, current and power of GBPG were measured as $2 \mathrm{~V}, 135 \mathrm{nA}$ and $45 \mathrm{nW}$, respectively. The average amplitude of the voltage, current and power of GPTG were measured as $4 \mathrm{~V}, 150 \mathrm{nA}$ and $83 \mathrm{nW}$, respectively. Similarly, the integrated GHSPS showed the superimposed effect and the average amplitude of the voltage, current and power of were $6 \mathrm{~V}, 280 \mathrm{nA}$ and $172 \mathrm{nW}$, respectively. Superposition can be successfully implemented to augment the electrical output based on the principles of piezoelectricity and triboelectricity. In addition, a table comparing the present device with others from the literature in terms of electrical output (peak voltage, current, power), material systems (substrate) and the differentiable characteristics is included in Table 1. The distinctive advantage of the hybrid system is primarily attributed to synergetic and efficient collection of the biomechanical energy, piezoelectrically and triboelectrically. Furthermore, the main difference between the proposed GHSPS and previously reported hybrid system [39] lies on the simplicity of structural construction, i.e., only the planar substrates are required as compared to the curved and roll-over configuration [39].

In order to quantitatively monitoring the human induced strains as actuated by muscle motions and the ambient wind blowing effect, the proposed highly transparent and stretchable GHSPS was attached to the neck by an adhesive tape (Figure 5a) and was placed on top of the cloth fabrics of chest (Figure 5b). Human induced motions can be monitored in an easy and self-powered manner. For example, the distinct muscle movements include the trachea for breathing and esophagus for drinking and eating can be individually monitored as various patterns. In Figure $5 \mathrm{c}$, the repeatedly expanding/contracting deformation of the skins associated with the trachea (breathing) and esophagus (drinking and eating) can be discriminatively differentiated by the proposed GHSPS. Furthermore, the output voltage of various human motions including, breathing, drinking, and eating could be measured as $\sim 0.8 \mathrm{~V} / 1.74 \mathrm{~V} / 5.32 \mathrm{~V}$ respectively. On the other hand, in Figure 5d, a kind of smart cloth was demonstrated which the varying wind speed can be effectively detected by the GHSPS as firmly attached on cloth fabric. A fan with an inner diameter of $45 \mathrm{~mm}$ was placed in front of chest, and the flow velocity was measured with the commercial wind meter (TES-1340). Output voltage under various air-flow velocities of $0 \mathrm{~m} / \mathrm{s}, 5.21 \mathrm{~m} / \mathrm{s}$ and $11.3 \mathrm{~m} / \mathrm{s}$ were measured as $\sim 0 \mathrm{~V} / 1.74 \mathrm{~V} / 5.32 \mathrm{~V}$ respectively. As a demonstration, the GHSPS-attached cloth exhibits 


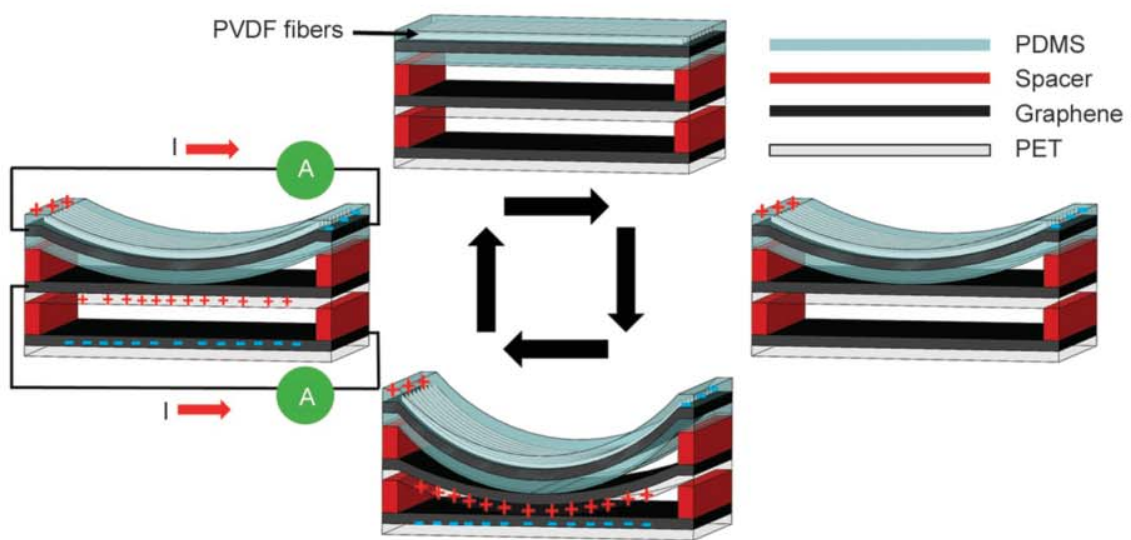

a)

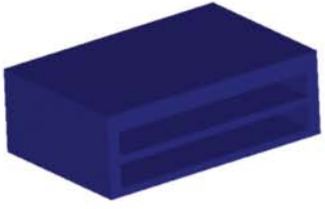

0.000

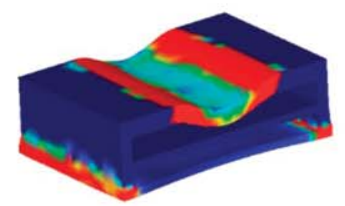

1.67

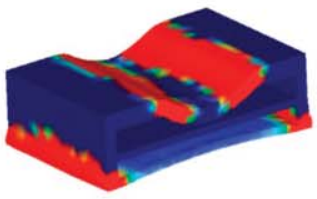

3.33

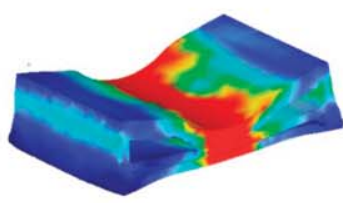

5.00

b)
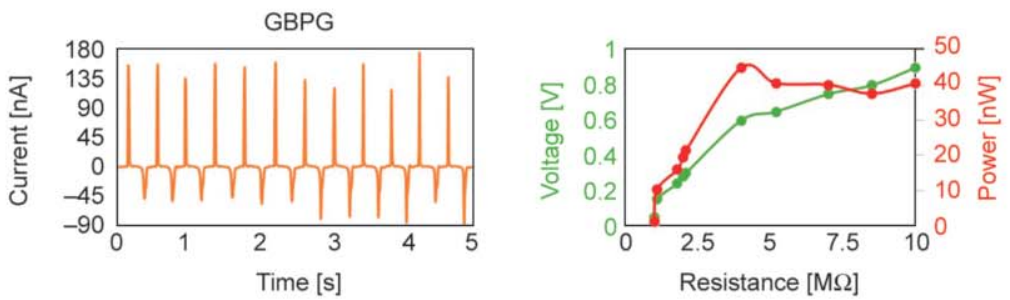

c)
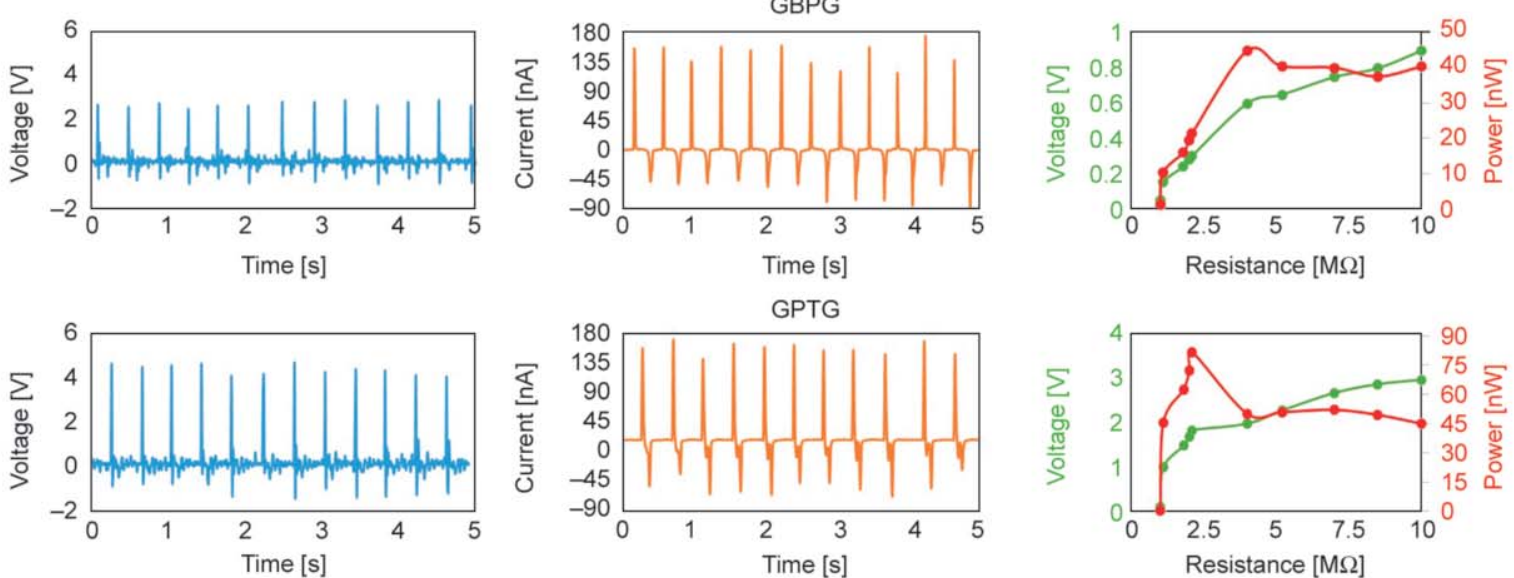

d)
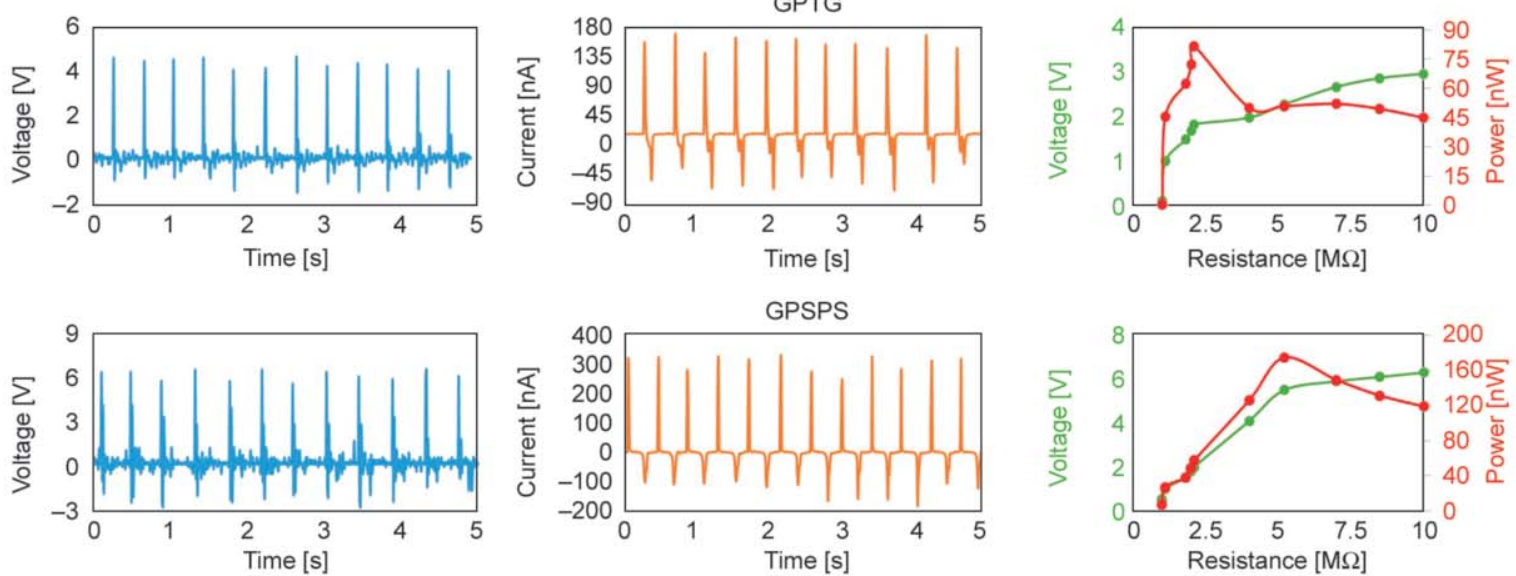

Figure 4. (a) Working mechanism of the proposed GHSPS device, which both piezoelectric (GBPG) and triboelectric (GPTG) charge generation and electricity generation process can be described by a combined cycle of the compression/deformation/contact/release processes. (b) The numerical simulation for GHSPS under the progression of compression and deformation. The PET-graphene mounted piezoelectric PVDF fibers were firstly bended and generates the electric potential via the effect of piezoelectricity. Followed by the triboelectric contact process of rubbing layers of graphene and PET respectively. The commercially available large plastic finite element software DEFORM 3D was used for simulating the large substrate deformation, and the center of the symmetrical cross section of the work piece had the maximum stress value $\sim 5 \mathrm{MPa}$. (c) The open-circuit voltage, short-circuit current, and output power of the piezoelectric device of GBPG with accumulated displacement $\sim 0.7 \mathrm{~mm}$ (corresponds to effective strain of 0.1 ) are measured as $\sim 2 \mathrm{~V} / 135 \mathrm{nA} / 45 \mathrm{nW}$ respectively. (d) The open-circuit voltage, short-circuit current, and output power of the triboelectric generators (GPTG) with graphene and PET contact/release process of accumulated displacement $\sim 0.35 \mathrm{~mm}$ are measured as $\sim 4 \mathrm{~V} / 150 \mathrm{nA} / 83 \mathrm{nW}$ respectively. (e) The superposition of opencircuit voltage, short-circuit current, and output power of the integrated GHSPS are measured as $\sim 6 \mathrm{~V} / 280 \mathrm{nA} / 172 \mathrm{nW}$ respectively. 
Table 1. Performance comparison

\begin{tabular}{|c|c|c|c|c|c|}
\hline & GHSPS in this paper & Ref. [34] & Ref. [35] & Ref. [39] & Ref [26] \\
\hline Peak voltage & $6 \mathrm{~V}$ & $1.8 \mathrm{~V}$ & $5 \mathrm{~V}$ & $100 \mathrm{~V}$ & $0.5 \mathrm{~V}$ \\
\hline Peak current & $280 \mathrm{nA}$ & $140 \mathrm{nA}$ & $0.5 \mu \mathrm{A}$ & $4 \mu \mathrm{A}$ & $0.3 \mu \mathrm{A}$ \\
\hline Power & $172 \mathrm{nW}$ & $45 \mathrm{nW}$ & $2.5 \mu \mathrm{W}$ & $450 \mathrm{nW}$ & $0.2 \mu \mathrm{W}$ \\
\hline $\begin{array}{c}\text { Displacement } \\
\text { Strain } \\
\text { Stress } \\
\text { Frequency }\end{array}$ & $\begin{array}{c}0.35 \mathrm{~mm} \\
0.1 \\
5 \mathrm{MPa} \\
2 \mathrm{~Hz}\end{array}$ & $\begin{array}{c}\text { NA } \\
0.576 \\
0.934 \mathrm{MPa} \\
4 \mathrm{~Hz}\end{array}$ & $\begin{array}{l}\text { NA } \\
\text { NA } \\
\text { NA } \\
\text { NA }\end{array}$ & $\begin{array}{c}\mathrm{NA} \\
0.2 \\
\mathrm{NA} \\
2.5 \mathrm{~Hz}\end{array}$ & $\begin{array}{l}\text { NA } \\
\text { NA } \\
\text { NA } \\
10 \mathrm{~Hz}\end{array}$ \\
\hline $\begin{array}{c}\text { Materials system } \\
\text { Substrate }\end{array}$ & $\begin{array}{c}\text { PVDF fibers/ } \\
\text { graphene-PET } \\
\text { ABS }\end{array}$ & $\begin{array}{c}\text { PVDF fibers/ } \\
\text { graphene-piezoelectric } \\
\text { PET }\end{array}$ & \begin{tabular}{|c|}
$\mathrm{ZnO} /$ \\
graphene-piezoelectric \\
PET \\
\end{tabular} & $\begin{array}{l}\text { PVDF fibers/ } \\
\text { Cu-PTFE } \\
\text { TPE }\end{array}$ & $\begin{array}{c}\mathrm{P}(\mathrm{VDF}-\mathrm{TrFE}) \\
\mathrm{BaTiO}_{3} \\
\mathrm{NA}\end{array}$ \\
\hline Characteristic & $\begin{array}{l}\text { Graphene-based } \\
\text { piezoelectric- } \\
\text { triboelectric generator, } \\
\text { highly transparent \& } \\
\text { structurally flexible }\end{array}$ & Transparent \& flexible & $\begin{array}{l}\text { Transparent \& high } \\
\text { mechanical elasticity }\end{array}$ & $\begin{array}{c}\text { PCB-based } \\
\text { piezoelectric - } \\
\text { triboelectric/ } \\
\text { Structurally robust }\end{array}$ & $\begin{array}{l}\text { Piezoelectric/coa } \\
\text { ted with a thin } \\
\text { gold layer highly } \\
\text { flexible \& easy } \\
\text { processing }\end{array}$ \\
\hline
\end{tabular}

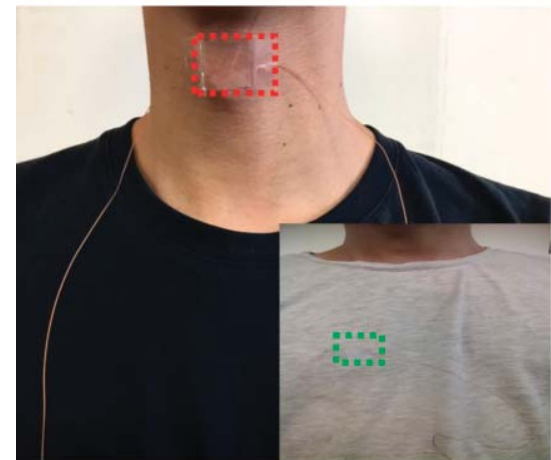

a)

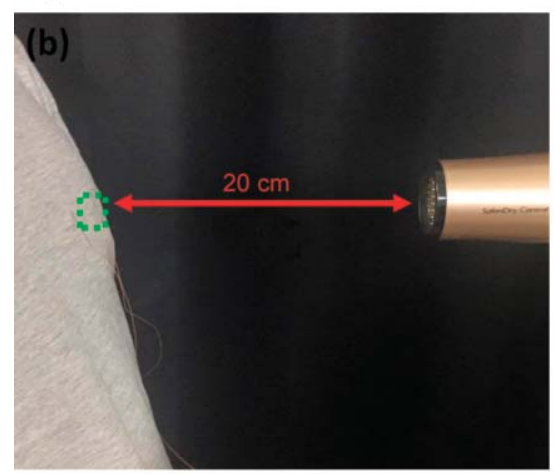

b)
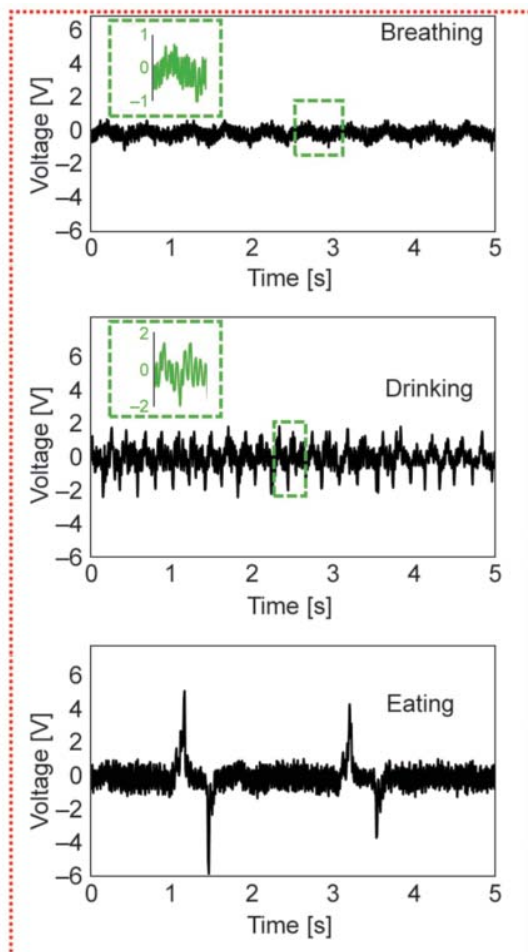

c)
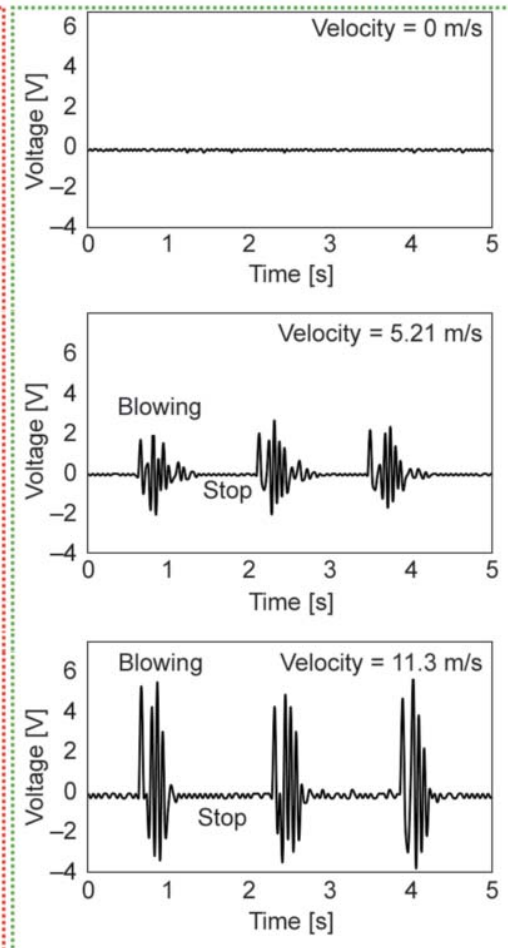

d)

Figure 5. Monitoring of muscle movement and wind blowing motion induced strains. Initially the stretchable and transparent GHSPS is adhesively attached to the (a) neck and the cloth fabrics on top of the chest. (b) The fan was placed at a distance $\sim 20 \mathrm{~cm}$ from the chest. (c) Output voltage change of the GHSPS versus time, during breathing, drinking, and eating, can be recorded and drastically different patterns can be monitored and measured as $\sim 0.8 \mathrm{~V} / 1.74 \mathrm{~V} / 5.32 \mathrm{~V}$ respectively. (d) Output voltage under various air-flow velocities, $0 \mathrm{~m} / \mathrm{s}, 5.21 \mathrm{~m} / \mathrm{s}$ and $11.3 \mathrm{~m} / \mathrm{s}$ were measured as $\sim 0 \mathrm{~V} / 1.74 \mathrm{~V} / 5.32 \mathrm{~V}$ respectively.

super-flexible and excellent conformability such that the dual functionality of a self-powered sensor and an energy harvesting device was successfully implemented under the wind blowing motion. We believe that this is a landmark progress for piezoelectric/triboelectric hybrid type self-powered sensors.

\section{Conclusions}

In summary, a fully packaged graphene and piezoelectric fibers is demonstrated with the excellent functionality of highly flexible and transparent features. The fabricated GHSPS consists of approximately five hundred piezoelectric PVDF fibers in parallel configura- 
tion as deposited via NFES technique. Utilizing both principles of triboelectricity/piezoelectricity, tiny mechanical energy can be converted into electricity with fully integration of a single layer graphene and aligned PVDF fibers. The hybridized structure of GBPG (piezoelectric) and GPTG (triboelectric) can generate output voltage/current about $6 \mathrm{~V} / 280 \mathrm{nA}$ under mechanical stress $\sim 5 \mathrm{MPa}$. Additionally, various human motions include breathing, drinking, and eating can be detected in a self-powered manner. The measurement results effectively distinguish with different power output. Furthermore, the fabricated device is attached to the cloth fabrics as a self-powered sensor to detect the effect of air-flow velocities in the range of $0-11.3 \mathrm{~m} / \mathrm{s}$. Due to the utilization of transparent materials of graphene electrode, the GHSPS exhibits a high optical transmittance of $\sim 87 \%$ for a single layer graphene GHSPS and $\sim 82 \%$ for two layer graphene GHSPS. Finally, the proposed manufacture method is straightforward, uncomplicated, low-cost and easily scale-up without sacrificing the advantages of structural durability and mechanical flexibility. The hybridized GHSPS will provide a framework to realize a ubiquitous application of self-powered energy systems.

\section{References}

[1] Rozhkov A. V., Giavaras G., Bliokh Y. P., Freilikher V., Nori F.: Electronic properties of mesoscopic graphene structures: Charge confinement and control of spin and charge transport. Physics Reports, 503, 77-114 (2011). https://doi.org/10.1016/j.physrep.2011.02.002

[2] Singh V., Joung D., Zhai L., Das S., Khondaker S. I., Seal S.: Graphene based materials: Past, present and future. Progress in Materials Science, 56, 1178-1271 (2011). https://doi.org/10.1016/j.pmatsci.2011.03.003

[3] Balandin A. A., Ghosh S., Bao W., Calizo I., Teweldebrhan D., Miao F., Lau C. N.: Superior thermal conductivity of single-layer graphene. Nano Letters, 8, 902 907 (2008).

https://doi.org/10.1021/n10731872

[4] Hwang J. O., Park J. S., Choi D. S., Kim J. Y., Lee S. H., Lee K. E., Kim Y-H., Song M. H., Yoo S., Kim S. O.: Workfunction-tunable, $N$-doped reduced graphene transparent electrodes for high-performance polymer lightemitting diodes. ACS Nano, 6, 159-167 (2012). https://doi.org/10.1021/nn203176u

[5] Eda G., Fanchini G., Chhowalla M.: Large-area ultrathin films of reduced graphene oxide as a transparent and flexible electronic material. Nature Nanotechnology, 3, 270-274 (2008).

https://doi.org/10.1038/nnano.2008.83
[6] Wang X., Zhi L., Müllen K.: Transparent, conductive graphene electrodes for dye-sensitized solar cells. Nano Letters, 8, 323-327 (2008). https://doi.org/10.1021/n1072838r

[7] Li X., Cai W., An J., Kim S., Nah J., Yang D., Piner R., Velamakanni A., Jung I., Tutuc E., Banerjee S. K., Colombo L., Ruoff R. S.: Large-area synthesis of high-quality and uniform graphene films on copper foils. Science, 324, 1312-1314 (2009).

https://doi.org/10.1126/science.1171245

[8] Kumar B., Lee K. Y., Park H-K., Chae S. J., Lee H., Kim S-W.: Controlled growth of semiconducting nanowire, nanowall, and hybrid nanostructures on graphene for piezoelectric nanogenerators. ACS Nano, 5, $4197-$ 4204 (2011). https://doi.org/10.1021/nn200942s

[9] Bae S-H., Kahya O., Sharma B. K., Kwon J., Cho H. J., Ozyilmaz B., Ahn J-H.: Graphene-P(VDF-TrFE) multilayer film for flexible applications. ACS Nano, 7, 3130 3138 (2013).

https://doi.org/10.1021/nn400848j

[10] Kwon J., Seung W., Sharma B. K., Kim S-W., Ahn J-H.: A high performance PZT ribbon-based nanogenerator using graphene transparent electrodes. Energy and Environmental Science, 5, 8970-8975 (2012). https://doi.org/10.1039/C2EE22251E

[11] Aubrecht G. J.: Energy: Physical, environmental and social impact. Pearson Education, London (2006).

[12] Wang Z. L.: Self-powered nanosensors and nanosystems. Advanced Materials, 24, 280-285 (2011). https://doi.org/10.1002/adma.201102958

[13] Paradiso J., Dutta P., Gellersen H., Schooler E.: Guest editors' introduction: Smart energy systems. IEEE Pervasive Computing, 10, 11-12 (2005). https://doi.org/10.1109/MPRV.2011.4

[14] Wang Z. L., Song J.: Piezoelectric nanogenerators based on zinc oxide nanowire arrays. Science, 312, 242-246 (2006). https://doi.org/10.1126/science.1124005

[15] Beeby S. P., Torah R. N., Tudor M. J., Glynne-Jones P., O’Donnell T., Saha C. R., Roy S.: A micro electromagnetic generator for vibration energy harvesting. Journal of Micromechanics and Microengineering, 17, 12571265 (2007). https://doi.org/10.1088/0960-1317/17/7/007

[16] Wang X., Wang S., Yang Y., Wang Z. L.: Hybridized electromagnetic-triboelectric nanogenerator for scavenging air-flow energy to sustainably power temperature sensors. ACS Nano, 9, 4553-4562 (2015). https://doi.org/10.1021/acsnano.5b01187

[17] Yang R., Qin Y., Li C., Zhu G., Wang Z. L.: Converting biomechanical energy into electricity by a muscle-movement-driven nanogenerator. Nano Letters, 9, 1201-1205 (2009). https://doi.org/10.1021/n1803904b

[18] Xu C., Wang X., Wang Z. L.: Nanowire structured hybrid cell for concurrently scavenging solar and mechanical energies. Journal of the American Chemical Society, 131, 5866-5872 (2009). https://doi.org/10.1021/ja810158x 
[19] Fuh Y-K., Chen P-C., Huang Z-M., Ho H-C.: Self-powered sensing elements based on direct-write, highly flexible piezoelectric polymeric nano/microfibers. Nano Energy, 11, 671-677 (2015).

https://doi.org/10.1016/j.nanoen.2014.10.038

[20] Cui J.: Zinc oxide nanowires. Materials Characterization, 64, 43-52 (2012). https://doi.org/10.1016/j.matchar.2011.11.017

[21] Chang C., Tran V. H., Wang J., Fuh Y-K., Lin L.: Directwrite piezoelectric polymeric nanogenerator with high energy conversion efficiency. Nano Letters, 10, 726 731 (2010).

https://doi.org/10.1021/n19040719

[22] Fuh Y-K., Chen P-C., Ho H-C., Huang Z-M., Li S-C.: All-direction energy harvester based on nano/micro fibers as flexible and stretchable sensors for human motion detection. RCS Advances, 5, 67787-67794 (2015). https://doi.org/10.1039/c5ra00275c

[23] Fuh Y-K., Chen S-Y., Ye J-C.: Massively parallel aligned microfibers-based harvester deposited via in situ, oriented poled near-field electrospinning. Applied Physics Letters, 103, 033114/1-033114/4 (2013). https://doi.org/10.1063/1.4813909

[24] Shen Y., Chen L., Jiang S., Ding Y., Xu W., Hou H.: Electrospun nanofiber reinforced all-organic PVDF/PI tough composites and their dielectric permittivity. Materials Letters, 160, 515-517 (2015). https://doi.org/10.1016/j.matlet.2015.08.019

[25] Nunes-Pereira J., Sencadas V., Correia V., Rocha J. G., Lanceros-Méndez S.: Energy harvesting performance of piezoelectric electrospun polymer fibers and polymer/ceramic composites. Sensors and Actuators A: Physical, 196, 55-62 (2013).

https://doi.org/10.1016/j.sna.2013.03.023

[26] Nunes-Pereira J., Sencadas V., Correia V., Cardoso V. F., Han W., Rocha J. G., Lanceros-Méndez S.: Energy harvesting performance of $\mathrm{BaTiO}_{3} /$ poly(vinylidene fluoride-trifluoroethylene) spin coated nanocomposites. Composites Part B: Engineering, 72, 130-136 (2015). https://doi.org/10.1016/j.compositesb.2014.12.001

[27] Fuh Y-K., Ye J-C., Chen P-C., Ho H-C., Huang Z-M.: Hybrid energy harvester consisting of piezoelectric fibers with largely enhanced $20 \mathrm{~V}$ for wearable and muscledriven applications. ACS Applied Materials Interfaces, 7, 16923-16931 (2015). https://doi.org/10.1021/acsami.5b03955

[28] Sato S., Yamada K., Inagaki N.: System for simultaneously monitoring heart and breathing rate in mice using a piezoelectric transducer. Medical and Biological Engineering and Computing, 44, 353-362 (2006). https://doi.org/10.1007/s11517-006-0047-z

[29] Kanik M., Say M. G., Daglar B., Yavuz A. F., Dolas M. H., El-Ashry M. M., Bayindir M.: A motion- and soundactivated, 3D-printed, chalcogenide-based triboelectric nanogenerator. Advanced Materials, 27, 2367-2376 (2015).

https://doi.org/10.1002/adma.201405944
[30] Huang T., Wang C., Yu H., Wang H., Zhang Q., Zhu M.: Human walking-driven wearable all-fiber triboelectric nanogenerator containing electrospun polyvinylidene fluoride piezoelectric nanofibers. Nano Energy, 14, 226235 (2015).

https://doi.org/10.1016/j.nanoen.2015.01.038

[31] Li Z., Wang Z. L.: Air/liquid-pressure and heartbeatdriven flexible fiber nanogenerators as a micro/nanopower source or diagnostic sensor. Advanced Materials, 23, 84-89 (2011). https://doi.org/10.1002/adma.201003161

[32] Hwang B-U., Lee J-H., Trung T. Q., Roh E., Kim D-I., Kim S-W., Lee N-E.: Transparent stretchable self-powered patchable sensor platform with ultrasensitive recognition of human activities. ACS Nano, 9, 8801-8810 (2015). https://doi.org/10.1021/acsnano.5b01835

[33] Liang Q., Yan X., Gu Y., Zhang K., Liang M., Lu S., Zheng X., Zhang Y.: Highly transparent triboelectric nanogenerator for harvesting water-related energy reinforced by antireflection coating. Scientific Reports, 5, 9080/1-9080/7 (2015). https://doi.org/10.1038/srep09080

[34] Lee J-H., Lee K. Y., Gupta M. K., Kim T. Y., Lee D-Y., Oh J., Ryu C., Yoo W. J., Kang C-Y., Yoon S-J., Yoo JB., Kim S-W.: Highly stretchable piezoelectric-pyroelectric hybrid nanogenerator. Advanced Materials, 26, 765-769 (2014). https://doi.org/10.1002/adma.201303570

[35] Zi Y., Lin L., Wang J., Wang S., Chen J., Fan X., Yang P-K., Yi F., Wang Z. L.: Triboelectric-pyroelectric-piezoelectric hybrid cell for high-efficiency energy-harvesting and self-powered sensing. Advanced Materials, 27, 2340-2347 (2015). https://doi.org/10.1002/adma.201500121

[36] Wu H., Kong D., Ruan Z., Hsu P-C., Wang S., Yu Z., Carney T. J., Hu L., Fan S., Cui Y.: A transparent electrode based on a metal nanotrough network. Nature Nanotechnology, 8, 421-425 (2013). https://doi.org/10.1038/nnano.2013.84

[37] Fuh Y. K., Kuo C. C., Huang Z. M., Li S. C., Liu E. R.: A transparent and flexible graphene-piezoelectric fiber generator. Small, 14, 1875-1881 (2016). https://doi.org/10.1002/smll.201503605

[38] Kim S., Gupta M. K., Lee K. Y., Sohn A., Kim T. Y., Shin K-S., Kim D., Kim S. K., Lee K. H., Shin H-J., Kim DW., Kim S-W.: Transparent flexible graphene triboelectric nanogenerators. Advanced Materials, 26, 39183925 (2014). https://doi.org/10.1002/adma.201400172

[39] Fuh Y-K., Li S-C., Chen C-Y.: Piezoelectrically and triboelectrically hybridized self-powered sensor with applications to smart window and human motion detection. APL Materials, 5, 074202/1-074202/9 (2017). https://doi.org/10.1063/1.4978913 